\title{
Voice Controlled Car using Aurduino and Bluetooth Module
}

\author{
Telugu Maddileti, Manideep Jammigumpula, H.Jagadish Kumar, K.V Sai Sashank
}

\begin{abstract}
This project builds a voice controlled car that can be controlled by voice commands which reacts in accordance to the corresponding voice command. However noise and distance handling require future development. Simple voice commands like left, right, forward, back, stop are used to run the car. These commands are given to Bluetooth module via an android application. The Bluetooth module and control unit are combined to store and test the voice commands. When an instruction for the automobile (car) is identified, a command message is sent to Arduino UNO, the Microcontroller of the car by the Bluetooth device. This command is analyzed by the microcontroller and followed up. In the vehicle, Image processing can be utilized to become aware of the shade and the obstacles. This work has been limited to the ZigBee system in the short-range (100mts range), and is linked to the car over long distance via long-range modules.
\end{abstract}

Keywords: Arduino UNO, Bluetooth module, Image processing, zigbee system, speech recognition.

\section{INTRODUCTION}

This project is related to a voice controlled car, which makes this project speech an important part of identifying commands and delivering them to the car via a wireless system. Identification of speech is also called as "automatic speech recognition (ASR)".This undertaking has numerous elements like, interaction between laptop and microprocessor, input and understanding of speech, Wireless numerical data distribution microprocessor to microprocessor, the control of several motors using microprocessors.

\section{A. Voice and Speech}

Voice is a sound which is produced by living beings. Voice uses airflow that comes from lungs. Air makes pressure over vocal folds which vibrate. Normally speech produces a whisper in our throat by using neck, chest, and abdomen this whisper becomes our speech. Our speech is unique for every person and also it helps other people to understand each

Revised Manuscript Received on December 30, 2019.

* Correspondence Author

Telugu Maddileti, Assistant Professor, ECM Department, Sreenidhi Institute of Science and Technology, Ghatekesar, Telangana-501301. E-mail:madhu14283@gmail.com.

Manideep .Jammigumpula, ECM Department, Sreenidhi Institute of Science and Technology, Ghatekesar, Telangana-501301 E-mail: manideepj2@gmail.com

H. Jagadish Kumar,, ECM Department, Sreenidhi Institute of Science and Technology, Ghatekesar, Telangana-501301 E-mail: jagadishkumarhiremath@gmail.com.

K.V. Sai Sashank, ECM Department, Sreenidhi Institute of Science and Technology, Ghatekesar, Telangana-501301 E-mail: saisashank.kv@gmail.com

(C) The Authors. Published by Blue Eyes Intelligence Engineering and Sciences Publication (BEIESP). This is an open access article under the CC BY-NC-ND license (http://creativecommons.org/licenses/by-nc-nd/4.0/) other's personality, mood and most importantly it helps people to communicate. Sounds propagate using mechanical waves for traveling around gases, liquids and solids. Mechanical waves transfer their energy from one medium to another medium while using vibration. Microphone is a hardware device which can convert analog input to a digital output. Digital data can be understood, modify and store by computers. Computers can recognize speech by using some complex algorithms and good dictionaries for these algorithms. Some systems use the Hidden Markov Model (HMM) and the Mel frequency cepstral coefficients (MFCC) techniques as well as the techniques of frequency spectral decomposition to use these two algorithms.

\section{B. Arduino Communication}

To communicate with Arduino we first need to install its free software from the internet and install. The software is very easy to use and installing it creates just one .uno files on the microprocessors these files confuse the user because there are many different files generating.

After installing Arduino it is ready to usage including dictionaries using dictionaries is very easy on the Arduino and Arduino does not requires any configuration setting when programming. User can use USB cable to connect Arduino and after that user can dump his code to Arduino far more easily and quickly than micro-controller.

\section{Bluetooth Communication}

Bluetooth is one of the popular devices to communicate in short range it is used on computers, cell phones, head phones and many other devices.

Bluetooth devices use 2.4 to $2.5 \mathrm{GHz}$ frequency to communicate with each other. Bluetooth standardized as IEEE 802.15.1 but then it changed that 802.15.1 Bluetooth's range is $2400-2483.5 \mathrm{MHz}$ approximately. Bluetooth devices generally use frequency-hopping spread spectrum communication technique to communicate with each other.

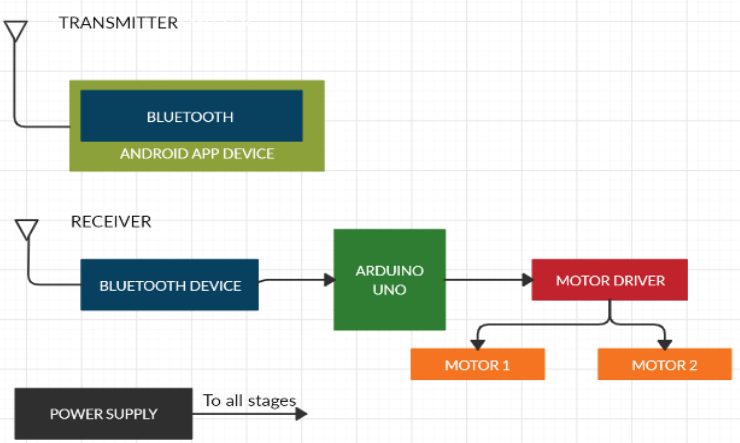

Figure 1: Block diagram of voice controlled car. 


\section{EXISTING SYSTEM}

The current systems are robots like line follower robot, edge averting robot, DTMF robot, gesture controlled robot. These type of robots are not efficient since they require more power to run, cost is also very high. In the existing system they don't use voice commands, making it not possible for physically handicapped people to drive.

The voice commands are interpreted via an offline server in real time. The commands are at once transmitted to the server directly by the means of a wired network. The car is built primarily on a platform based on a microcontroller.Some of the fields that can likewise be equally enhanced are the effect of the mouth-microphone range on the robotic, the overall performance (scope) of the robot and the effect of noise on the translation of speech to textual content.

In the existing system BitVoicer Server is used, it's a database for speech processing and automation synthesis. It was designed to make voice operation possible with simple gadgets having low processing power. Microcontrollers usually do not have enough storage and computing ability to perform sophisticated speech treatment and synthesis. By doing the tough work BitVoicer Server removes the consequences of these limitations so that the microcontroller can assign its key functionality to most of its origin sources.

\section{PROPOSED SYSTEM}

In this proposed device we perform a variety of research on control style variants for robots. It shows that it's feasible to study to successfully manipulate actual world objects with solely voice (human voice) as a control mechanism. The reason of this lookup is to provide simple robotic hardware architecture so that this shape can focal point on Bluetooth connection infrastructure. It is also beneficial for academic robotics due to the fact human beings can construct their personal robots with low cost.

When the app is operating in the system, a microphone on the mobile is used to identify user voice commands. Commands are interpreted and the program utilizes Google's speech-recognition software to translate voice to text within the app. The text will then be sent with the aid of Bluetooth to the receiver part.

The microcontroller Arduino UNO R3 has $32 \mathrm{kB}$ of ISP flash memory, $2 \mathrm{kB}$ of RAM and $1 \mathrm{kB}$ of EEPROM. The panel incorporates serial communication connectivity with UART, SPI and I2C. The MCU will operate at $16 \mathrm{MHz}$ clock speed.

The digital Arduino I / O pins 3, 4, 5 and 6 are programmed as output pins in this design. For serial communication with the Bluetooth unit, pins 0 and 1 of Arduino are used. Text obtained with the aid of Bluetooth is forwarded to Arduino UNO microcontroller panel by the usage of UART serial conversation protocol. Table 3.1 displays the voice commands used to monitor the robots and their functions.

Table 3.1: Voice command functions

\begin{tabular}{|c|l|}
\hline $\begin{array}{c}\text { Voice } \\
\text { Command }\end{array}$ & Function \\
\hline Forward & Bot moves forward \\
\hline Backward & Bot moves backward \\
\hline Right & $\begin{array}{l}\text { Bot turns right and carries on } \\
\text { riding }\end{array}$ \\
\hline
\end{tabular}

\begin{tabular}{|c|c|}
\hline Left & $\begin{array}{c}\text { Bot turns left and carries on } \\
\text { riding }\end{array}$ \\
\hline Stop & Bot stops moving \\
\hline
\end{tabular}

The voice commands to the robotic device are dispatched via Bluetooth with the aid of an Android device. These commands are received on the robotic device by using Bluetooth module set up on it. The motor driver circuit is used to manipulate the velocity of the car. The complete circuitry is powered by the usage of a $12 \mathrm{~V}$ rechargeable battery hooked up on the system.

\section{Testing}

Upon successfully pairing the device, open the app on the smart phone and press on the Bluetooth textual and emblematic pushbutton. The number of associated gadgets will now be shown. Select HC-05 from the listing to join the smart phone with HC-05 Bluetooth module on the receiver side. After successful connection, 'connected' will be displayed on the primary screen of Voice control app. Press the pushbutton with microphone image and a prompt will show up asking for voice commands.

- When it appears, voice instructions are detected via the app, which converts them into textual content and sends it to the receiver aspect wirelessly by using Bluetooth. On the receiving side, Arduino tests the text. If it is a matching string, it controls the moves of the robot in accordance to the description.

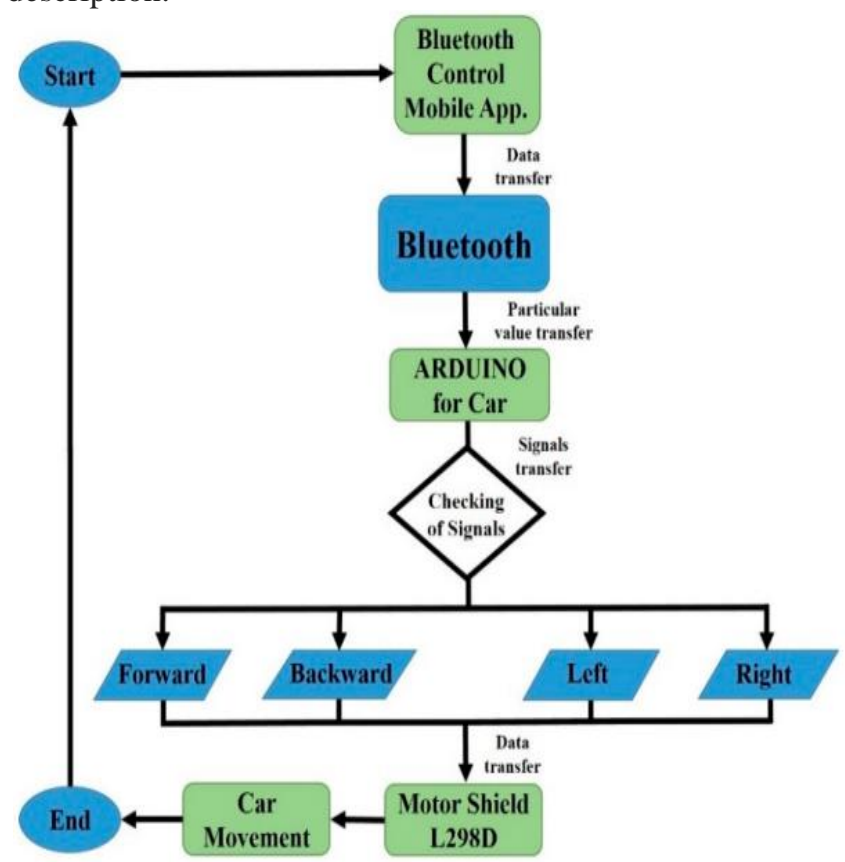

Figure 3.1.1: Flowchart of Voice controlled car

\section{RESULTS}

- Voice controlled car is working and all the functions are followed by the bot.

- Unlike DTMF robot, the car is connected to the mobile phone wirelessly making it comfortable to the user to control the car.

- The line follower robot moves only in a particular path, in case there is an obstacle in its path it won't move unless the obstacle is removed. 
- Various languages can be given as a voice input using Google's speech recognition technology rather than using an offline BitVoicer server.

The result will be shown in following figures.

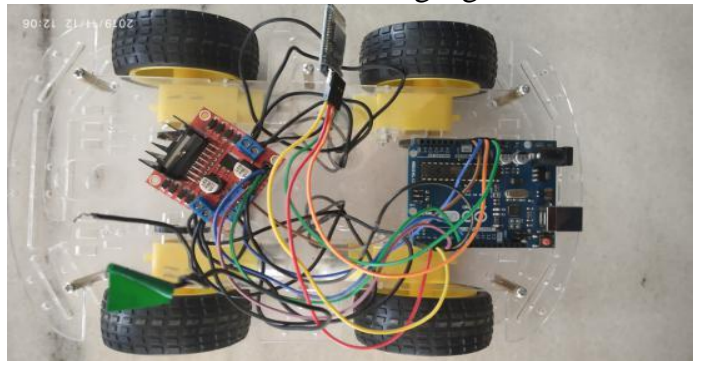

Figure 4.1:Voice controlled car (Top view)

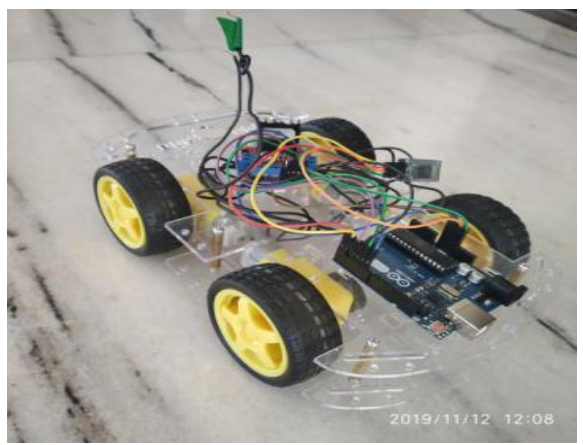

Figure 4.2:Voice controlled car (side view)

The following figures shows the Bluetooth connectivity to the Bluetooth module.

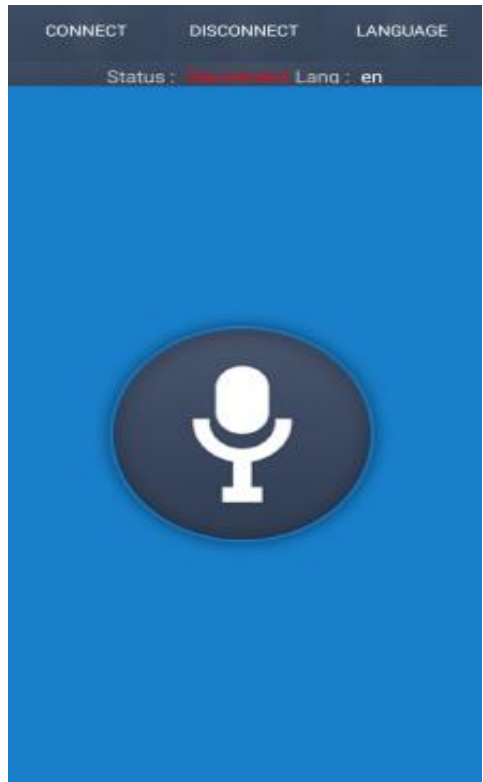

Figure 4.3: Status Disconnected

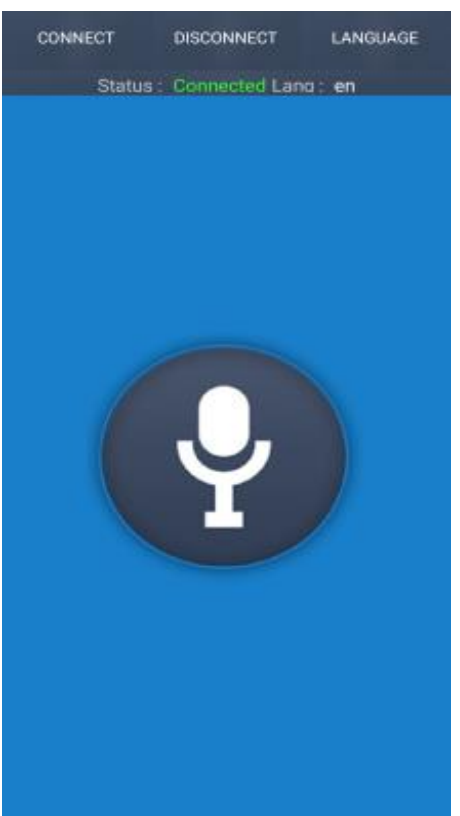

Figure 4.4: Status Connected

\section{FUTURE SCOPE}

This experiment was limited to a brief zigbee device (range 100 meters), and is linked to the car over long distance via long-range modules. Sleep and wake-up routines may be enforced for energy enhancement. In the vehicle, Image processing can be utilized to become aware of the shade and the obstacles. For additional proper service servo motors can be deployed.

A robot may include an automated targeting system to monitor the aim.

\section{CONCLUSION}

In this project the voice control was designed for a hoe assistant robot. The order of speech signals is automatically transmitted via a wired network to the server. The car is built primarily on a platform based on a microcontroller. Evaluation of the output of the original tests is carried out with promising implications.

Possible developments to feasible technologies in households, schools, vehicle networks and businesses are also addressed.

Several areas that may additionally be discussed are the impact of noise on speech to textual content translation. The accent of the speaker no longer affects the robotic activity because speech commands are interpreted using a cloud-based server that works independently of the speaker accent.

The use of renewable energy sources for robotic operation would not only increase the value of robotic energy, but would also be environmentally friendly. Solar cells can be a suitable power source to use.

The design of the robotic assistant is ideal for applications ranging from chemical manufacturing to comfortable home circumstances.

Accuracy of detecting a voice command correctly is found to be $75 \%$.

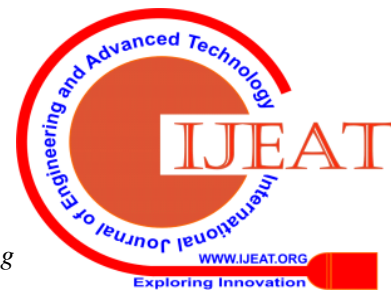




\section{REFERENCES}

1. https://create.arduino.cc/projecthub/Yug_Ajmera/

2. https://drive.google.com/drive/folders/0BwsV1jJYW9dndjZKaTBwakJ uOFk

3. https://www.instructables.com/id/

4. https://www.researchgate.net/publication/325722323_IJSRST173866_ Bluetooth_Remote_Controlled_Car_using_Arduino

5. https://ieeexplore.ieee.org/document/8093565

6. https://www.viralsciencecreativity.com/post/

7. https://nevonprojects.com/

8. https://www.hackster.io/Yug_Ajmera/

\section{AUTHORS PROFILE}

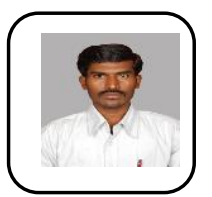

Mr.Telugu Maddileti is currently working as Assistant Professor in the department of Electronics and Computer at SNIST and Research Scholar at JNTUA, Ananthapuramu. He has a total of 14 years experience in academics. He completed B.Tech degree (Electronics and communication Engineering) in the year 2005 and M.Tech in 2009 from JNT University, Hyderabad. He is enthusiastic to work in the field of VLSI and Image Processing. His area of interest includes Low power VLSI domain, optimization of analog and mixed signal circuits in addition to digital system design using CAD tools. He has presented more than 15 International/National Technical Papers in journals \& conferences.

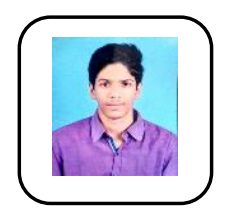

Manideep Jammigumpula pursuing his B.Tech degree from JNTU, Hyderabad. His areas of interest includes communications, image processing and IOT based designs and applications. He has Presented 4 International/National Technical Papers in journals \& conferences.

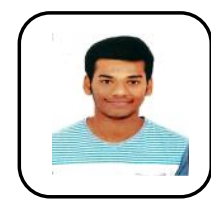

H.Jagadish Kumar pursuing his B.Tech degree from JNTU, Hyderabad. His areas of interest includes communications, image processing and IOT based designs and applications. He has Presented 4 International/National Technical Papers in journals \& conferences.

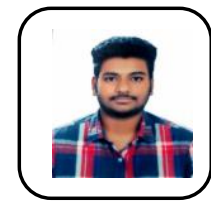

K.V.Sai Sashank pursuing his B.Tech degree from JNTU, Hyderabad. His areas of interest includes communications, image processing and IOT based designs and applications. He has Presented 4 International/National Technical Papers in journals \& conferences. 